\title{
Multiphase Periodic Very-Low-Frequency Emissions
}

\author{
Neil Brice ${ }^{1}$ \\ Contribution From the Radioscience Laboratory, Stanford University, Stanford, Calif.
}

(Received July 10, 1964; revised September 17, 1964)

\begin{abstract}
When periodic very-low-frequency (VLF) emissions of more than one set are observed concurrently (multiphase emissions) the observation of three sets symmetrically spaced is surprisingly frequent. Evidence is presented which suggests that two sets represent a transient situation, and that the strength of one emission may depend on the strength of, and the elasped time since the preceding emission. The data are interpreted as evidence of a relaxation phenomenon in the generation of discrete VLF emissions, and a qualitative explanation of the stability of symmetrical three-phase emissions is given.
\end{abstract}

\section{Introduction}

Discrete very-low-frequency (VLF) emissions which are observed at regular intervals and are called periodic emissions were first reported by Dinger [1957], who likened the sound of these emissions to that of "squeaky wagon wheels." Spectra of periodic emissions were published by Gallet [1959]; Pope and Campbell [1960]; and Brice [1962], while spectra of conjugate periodic emissions, in which the emission appeared alternately in opposite hemispheres, were published by Lokken et al. [1961]. Helliwell [1963] showed that, for the latter, the first emission observed coincided in time of occurrence and frequency range with the ninth hop of a whistler echo train and that the period of the emissions and the whistler two-hop group delay were the same. Many different "sets" of periodic emissions were found during the recording period and in all cases the agreement between the emission period and the whistler two-hop group delay was extremely good. Each emission was labeled by Helliwell [1963] according to the "set" (A, B, C, etc.) with a subscript denoting the number of emissions in the set which preceded it $(0,1,2$, etc.). Where a number of sets overlapped so that the sequence of emissions observed was, for example, $\mathrm{D}_{8}, \mathrm{E}_{7}, \mathrm{~F}_{2}, \mathrm{D}_{10}$, the emissions were referred to as "multiphase emissions." Helliwell [1963] suggested that, as emissions were triggered by whistlers, the emissions, echoing in the whistler mode, might trigger new emissions. A series of periodic emissions would thus be produced, for which the emission period was precisely the same as the whistler two-hop delay. Considerable additional evidence to support the hypothesis has been found by Helliwell and Brice [1964]. 1 Now at Faculty of Engineering, Carleton University, Ottawa, Ontario,

\section{Symmetrical Multiphase Emissions}

On occasion, periodic emissions are found for which, from a casual inspection, it might be suggested that the emission period was much less than the expected two-hop whistler group delay, spectra of an example being given in figure 1. However, the later emissions of figure 1 show that every third emission is noticeably stronger. This suggests that the stronger emissions constitute one set of periodic emissions, so that the true or "fundamental" period is three times the "apparent" period. The weaker emissions are members of two other sets, each set being displaced in time by one-third of the true emission period with respect to the other sets. Because of this symmetry, these emissions are called symmetrical multiphase emissions, those shown in figure 1 being symmetrical three-phase emissions. The emissions shown in figure 1 were first interpreted as symmetrical multiphase emissions by M. Trimpi [1962, private communication] of Stanford. Since that time, many additional examples of symmetrical three-phase emissions have been recognized, but symmetrical twophase emissions appear to be extremely rare. This suggests that the existence of three sets (or phases) of periodic emissions represents a relatively stable configuration and two phases relatively unstable. Strong support for this suggestion is provided by the periodic emissions recorded at two conjugate pairs of recording stations on 1 April 1963. A program of 3-hr continuous tape recordings of VLF phenomena at Byrd and Eights Stations in the Antarctic and Great Whale River and Quebec City in Canada was initiated by Stanford University. Periodic emissions were observed continuously from the start of one such recording schedule at 2300 UT 1 April 1963, until almost 0010 U'T, 2 April 1963. Spectra of single- 


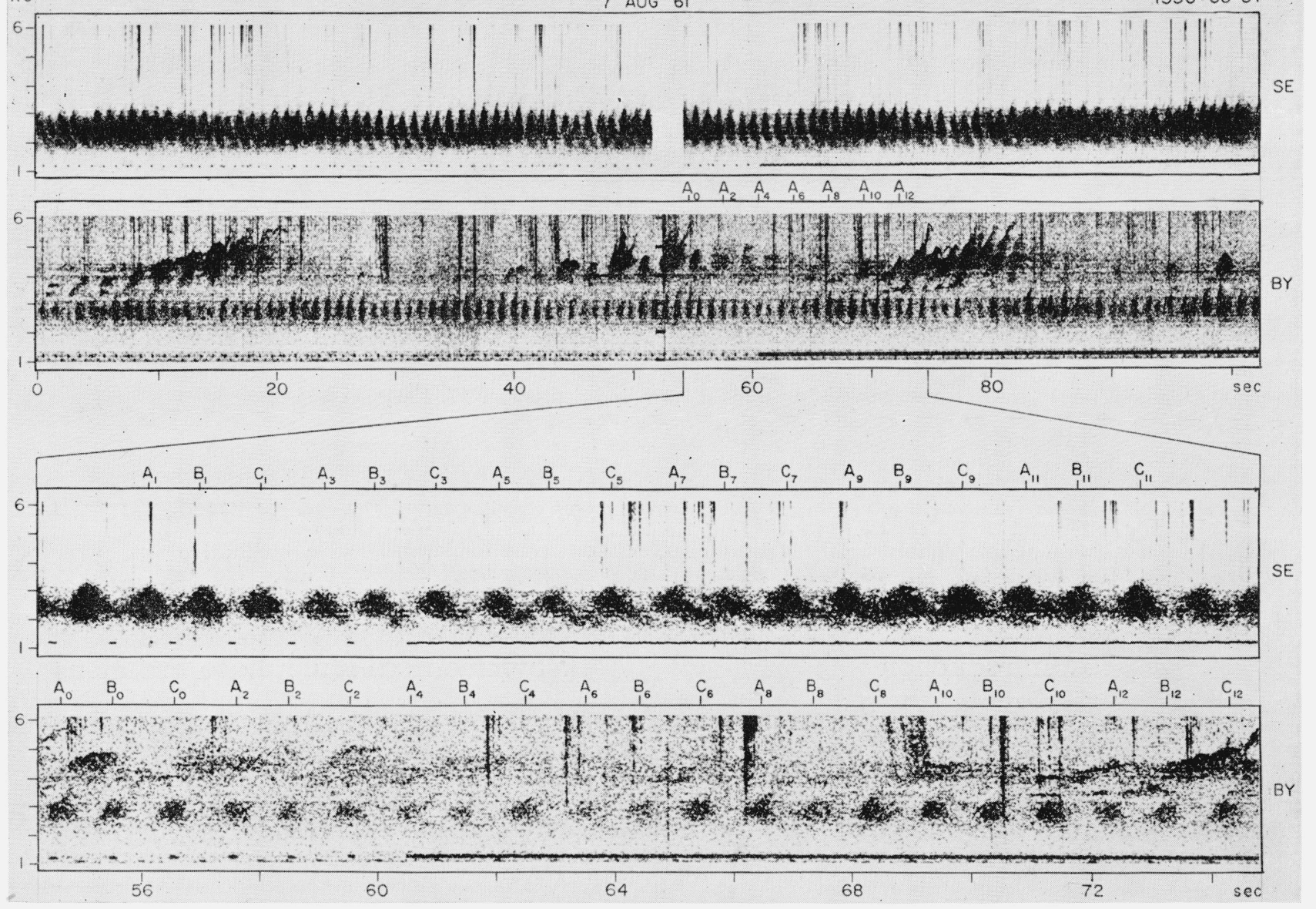

Figure 1. Spectra of three phase emissions recorded at Seattle, Washington and Byrd Station, Antarctica.

phase emissions recorded at all four stations are shown in figure 2, and spectra of three-phase emissions in figure 3 . These emissions were recognized as interesting by M. Trimpi [private communication] from aural analysis of tape recordings at Eights Station, Antarctica. Since the Antarctic tape recordings were not immediately available, analysis was made of the conjugate emissions recorded at Great Whale River in Canada. Figure 4 shows spectra of the Great Whale River recording from 2300 UT on 1 April 1963 to 0020 UT, 2 April 1963. From this figure, it is seen that the emissions are predominantly single-phase from 2300 to $2312 \mathrm{U} T$, then three-phase until 2320 U'T, single-phase until 2337 U'T, then three-phase until $2343 \mathrm{U} T$, and then single-phase until 0009 U'T, after which time no further emissions were observed. For the single phase emissions, the duration of an individual emission is much less than the spacing between emissions. It is expected then that the spectrum of the envelope of the single-phase emissions would show the fundamental emission period and several harmonics. For the three-phase emissions, however, the duration of the emissions is about the same as the spacing between them, so that the envelope of those emissions would be very nearly sinusoidal. The spectrum of this envelope would then show only a single frequency component, the period being just one-third of the fundamental period. These features are shown in figure 5, where the spectrum of the amplitude of the signals at $1.25 \mathrm{kc} / \mathrm{s}$ is shown. The transitions from single-phase to three-phase emissions are readily apparent. The spectrum shows initially the fundamental frequency (approximately $0.25 \mathrm{c} / \mathrm{s}$ corresponding to the fundamental emission period of about $4 \mathrm{sec}$ ) and several harmonics of this frequency. At the transition to three-phase emissions, the amplitude of the third harmonic is increased slightly while the fundamental and other harmonics disappear. The pattern observed in figure 5, single-phase, three-phase, then single, three, and finally single-phase corresponds with that found in figure 4. The comparative stability of the fundamental period, as is evident in figure 5 , is expected when the period is interpreted as being equal to the two-hop whistler-mode group delay for the path of propagation, since whistler group delays are usually constant for periods up to a few hours [Carpenter, 1960]. Also, the integral (3 to 1) relationship between the periods of the two types of emissions lends strong support to the inter- 

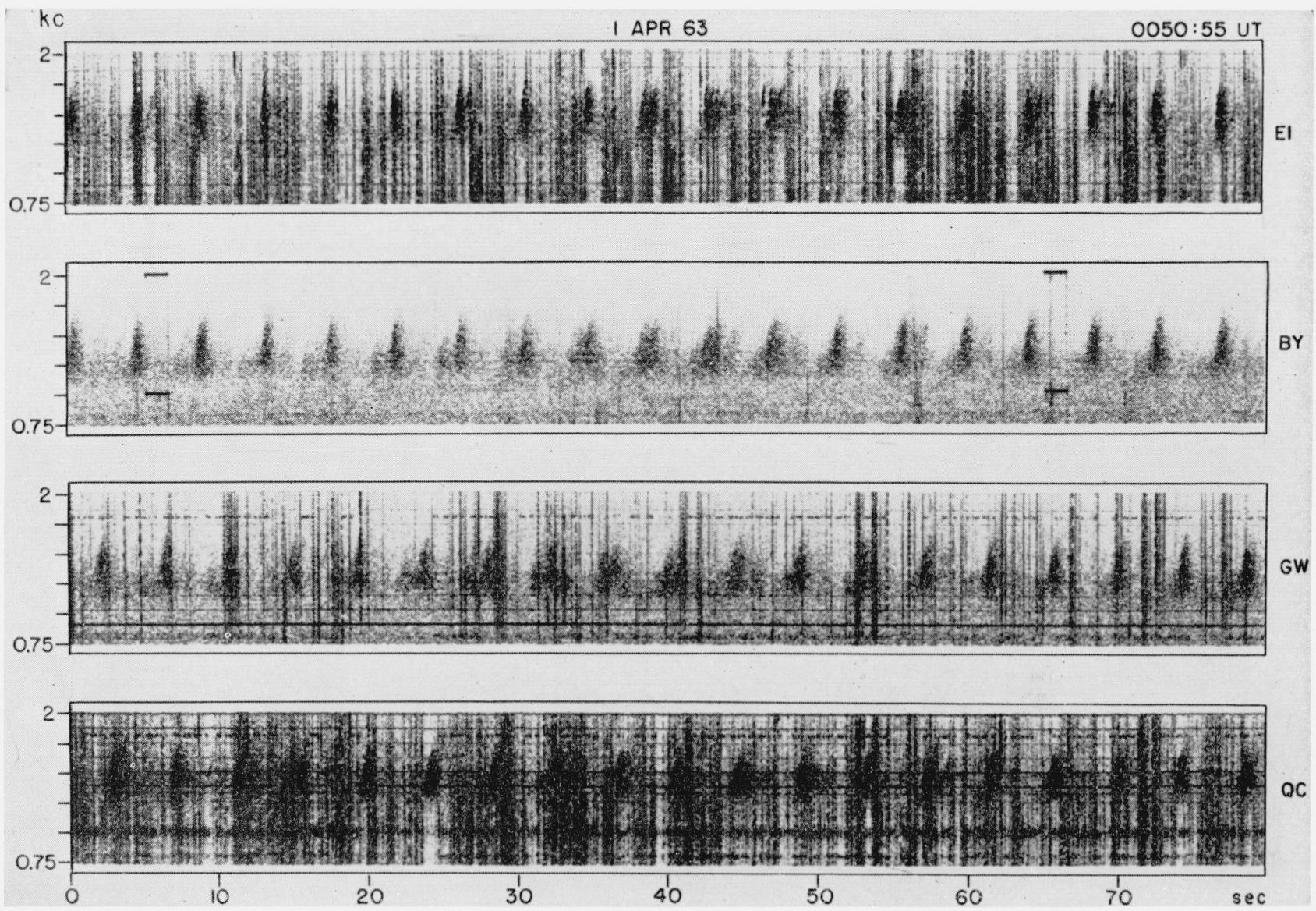

Figure 2. Single phase periodic emissions recorded at two pairs of magnetically conjugate locations.

pretation that these are, in fact, single-phase and symmetrical three-phase emissions. This integral relationship may also be verified from figure 6 , which shows two sections of the emissions in figure 4, shown with expanded time scales, illustrating single and three-phase emissions respectively.

It is apparent that the three-phase emissions of figure 6 show "amplitude modulation" with a period of about $30 \mathrm{sec}$, and examination of figure 4 shows that the amplitude modulation is in evidence throughout the period from 2330 to 2340 U'T.

\section{Two-Phase Emissions}

The emissions recorded during the time 2330 to 2342 U'T are of particular interest, sections being shown in figures 7 and 8 . These figures are enlargements of parts of figure 9, which shows the emissions recorded from 2331:20 to 2336:30. In these figures, the labels are intended to indicate the approximate position of the emission and should not be interpreted as precise measurements of the location of the maximum amplitude of the emission. In figure 7 , the first emission shown is labeled $\mathbf{A}_{0}$. For $10 \mathrm{~min}$ prior to this time, the emissions were simple singlephase periodic emissions. Following $A_{0}$ is $A_{2}\left(A_{1}\right.$ being observed in the opposite hemisphere) and then $\mathrm{A}_{4}$, with the spacing between the emissions corresponding to the fundamental emission period. Then, after about two-thirds of this period, the first emission of a new set, $B_{0}$, appears and is closely followed by $A_{6}$, then $B_{2}, A_{8}, B_{4}, A_{10}, B_{6}, B_{8}, B_{10}$. The emissions $B_{0}, B_{2}$, and $B_{4}$ show a systematic increase in amplitude, while $A_{6}, A_{8}$, and $A_{10}$ show a systematic decrease, and no emission is observed at the time when $A_{12}$ would be expected to appear. A dozen sequences showing essentially these same features were found amongst the emissions of figure 4. The only two-phase emissions with a different pattern are shown in figure 8. Here two differences are noted, in that the first emission of the new sequence, $\mathrm{C}_{0}$, appears about equidistant in time between $\mathrm{B}_{12}$ and $\mathrm{B}_{14}$ (instead of after twothirds of a period) and the new sequence, $\mathrm{C}$, does not take over, but disappears after $\mathrm{C}_{4}$ while the emissions of set B continue. In figure 9, the general pattern of the two-phase emissions is shown, with set $B$ taking over from set $A$, then set $C$ (the exception not taking over from $B$ ), then set D taking over from $\mathrm{B}, \mathrm{E}$ from $\mathrm{D}, \mathrm{F}$ from $\mathrm{E}$ and so on until set $J$ takes over from set I. Also observable in figure 9 is the amplitude modulation with the $30 \mathrm{sec}$ period referred to earlier, and it is noted that in 

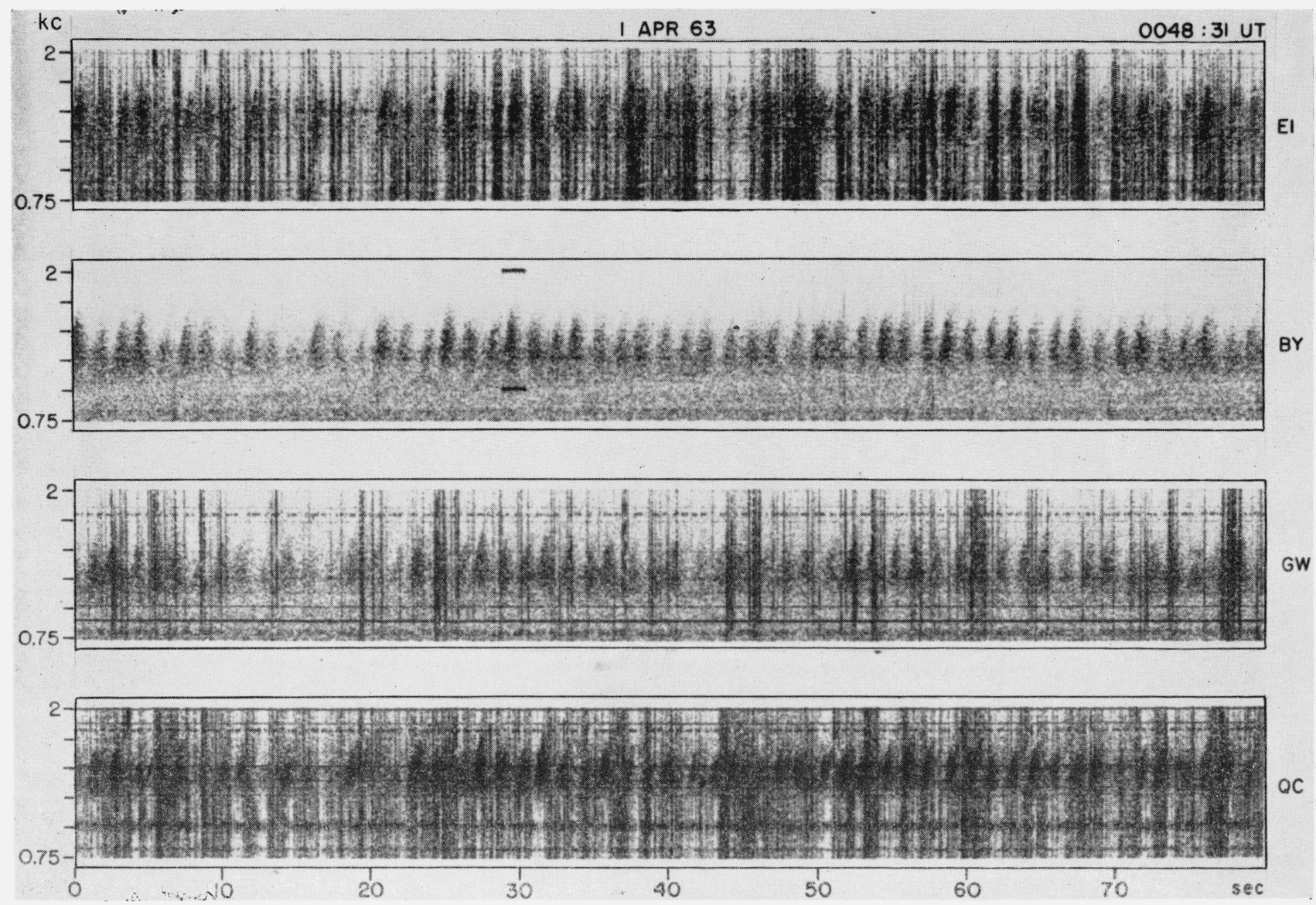

FIGURE 3. Symmetrical three phase emissions recorded in opposite hemispheres.
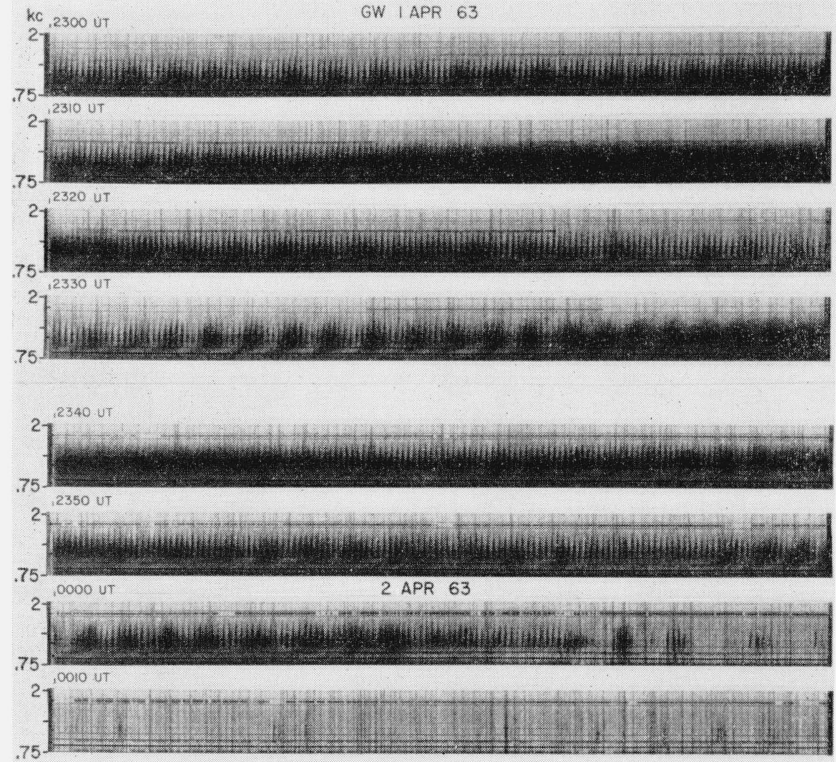

Figure 4. Periodic emissions with a duration of approximately $70 \mathrm{~min}$. every case the first emission of the new set appears when the average amplitude of the emissions is large.

These features are also apparent in figure 10, which shows the autocorrelation computed from the amplitude of the emissions in figure 9. The most noticeable features of the autocorrelation are the regular maxima and minima arising from the fundamental periodicity of the emissions. Also apparent is the variation of the "average" correlation coefficient, arising from the "amplitude modulation" of the emissions with $30 \mathrm{sec}$ period. The third feature of interest is the small bump which appears on the left-hand side of the main peak. The first small bump indicates the probability of seeing emission after two-thirds of the fundamental period, and this is small compared with the probability for one period. After one and two-thirds periods, the difference between the height of the bump and that of the main peak is less. This difference continually decreases until the probability of observing an emission after six and two-thirds times the fundamental period is greater than after seven periods. Thus the average time required for the emissions of the new set to take over is about $30 \mathrm{sec}$, the same as the period of the amplitude modulation. 


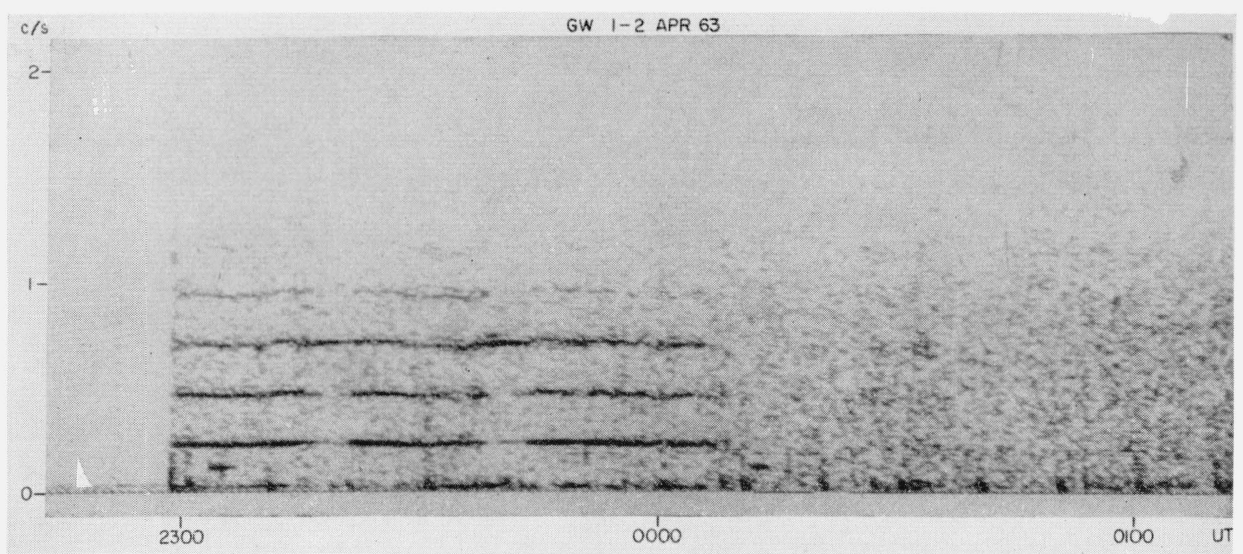

FIGURE 5. The fourier spectrum of the emission amplitude at $1.25 \mathrm{kc} / \mathrm{s}$ showing the fundamental periodicity of the emissions.
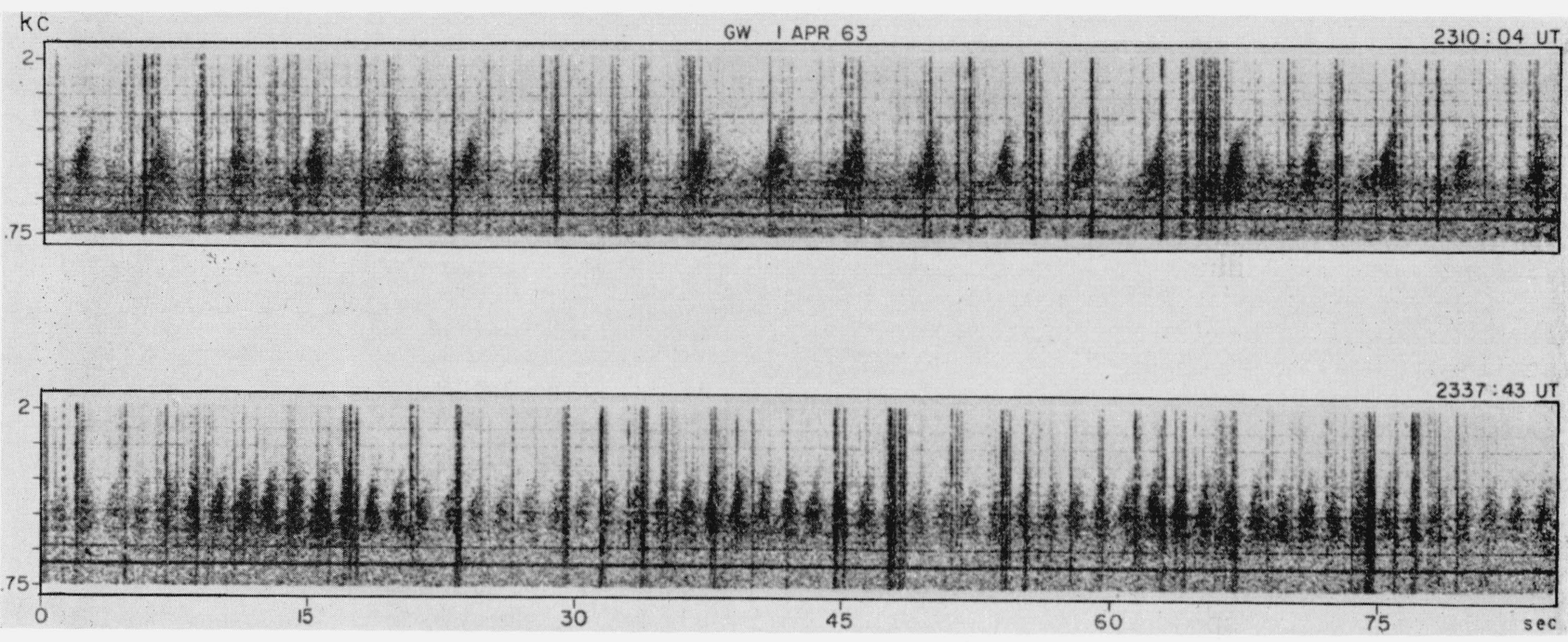

FIGURE 6. Single-and symmetrical three-phase emissions obtained during the same recording period and illustrating the integral (3 to 1) ratio of the emission periods.

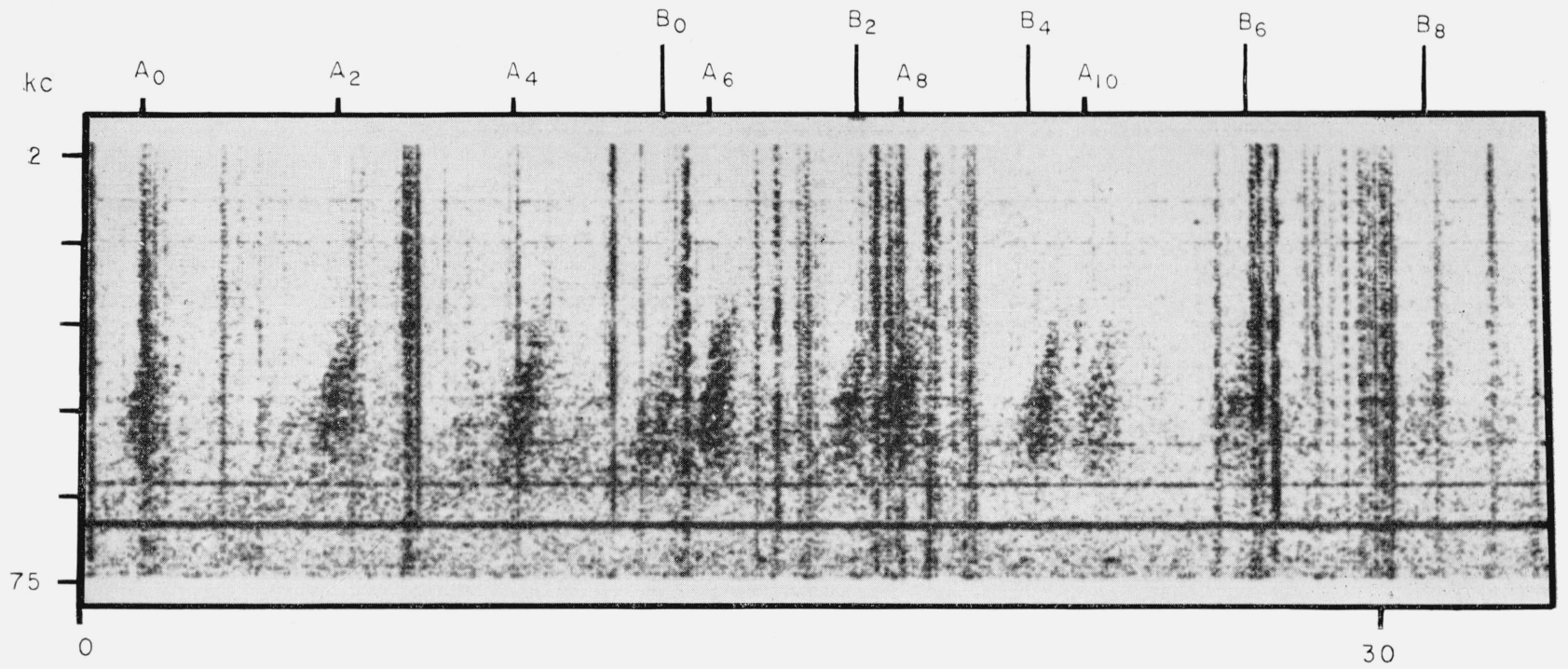

FIGURE 7. Growth and decay of sets of periodic emissions, showing that the emissions with the greatest "dead time" before them eventually dominate. 


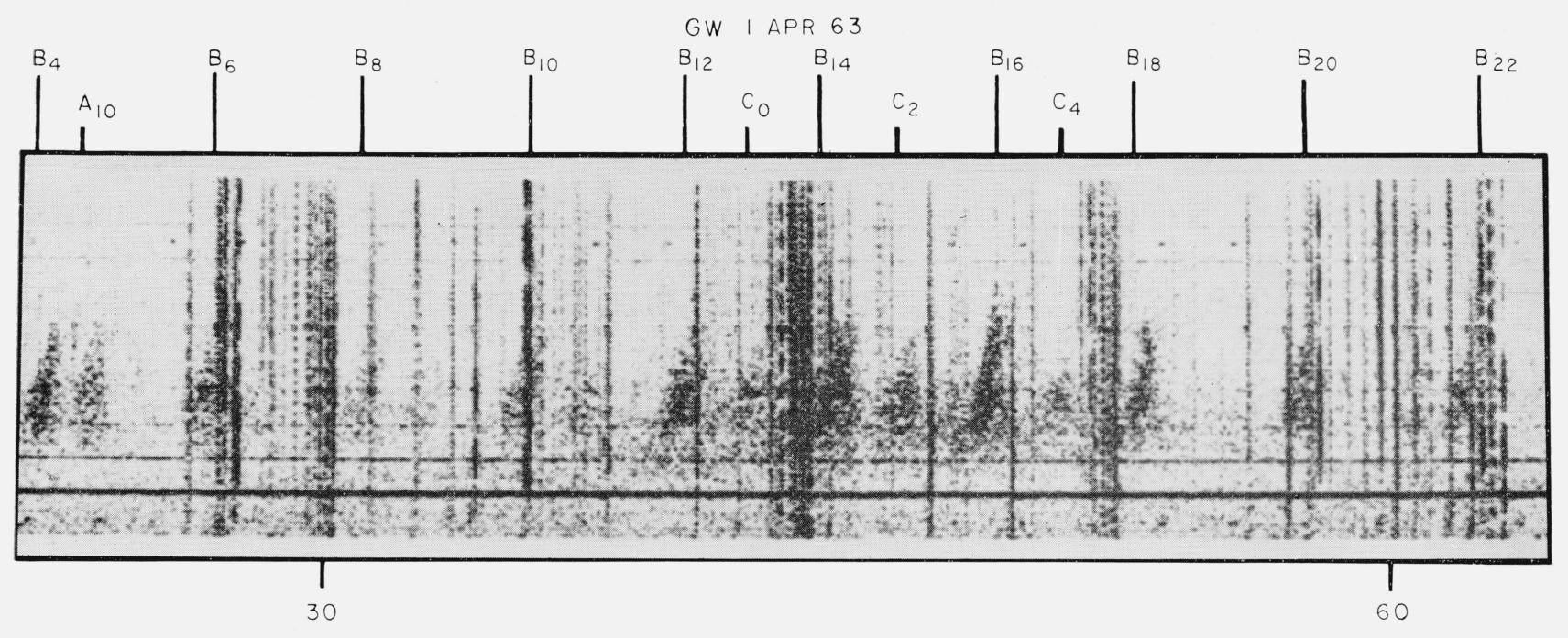

FiguRE 8. Two phase emissions illustrating that the strongest emission set survives while the weaker set decays.

\section{Analysis Techniques}

Figures 5 and 10 illustrate analysis techniques which have not previously been used for VLF emissions, and it is therefore of interest to describe these techniques briefly. The emissions were initially recorded on magnetic tape. To obtain figure 5 the tape was replayed and the signal out of the tape recorder was fed into a filter at $1.25 \mathrm{kc} / \mathrm{s}$ and then detected. The detected signal was then fed into a very slow speed tape recorder (several inches per hour) of the type commonly used for recording micropulsations. This tape was then replayed at 60 in. per second the "speed up" factor being almost 4100 (actually 4096), and the output signal fed into a conventional sonagraph spectrum analyzer. The 0 to $8 \mathrm{kc} / \mathrm{s}$ range of the analyzer becomes approximately 0 to $2 \mathrm{c} / \mathrm{s}$ when referred to the original recording of the emissions.

With a suitable selection of filter frequencies, this technique could be used for a field station periodic emission detector. Slow speed tape recordings made at field stations could be replayed at high speeds, the appearance of constant frequency tones indicating the presence of periodic emissions. Many hours of tape could be analyzed in a few seconds, and the periods of the emissions found by measuring the frequencies of the tones heard on playback.

To obtain figure 10, the tape recorder output was filtered and detected as described above. The amplitude of the detected signal was then sampled 25 times each 2 sec, using an analog-to-digital converter. The autocorrelation was then computed using an IBM 7090 computer. The regularity of the emissions may be judged from the fact that, using a sampling rate of 50 a second for single phase emissions, and two thousand samples, a correlation coefficient of 0.8 was obtained for a correlation delay of twice the emission period. This technique allows the emission period to be found to a high degree of accuracy, even when many sets of emissions are present and the number of individual emissions in each set is not large. Determination of the emission period with precision is necessary if the period is to be measured at different frequencies and the whistlermode nose frequency found and hence the magnetic field line path along which the emissions were generated [Smith and Carpenter, 1961; Brice, 1964a].

\section{Interpretation}

The important facts about the emissions of figure 3 analyzed above may be summarized as follows: for the two-phase emissions, the first emission of a new set never appears when the elapsed time since the observation of the previous emission is significantly less than half the fundamental period, and in all cases except one, this delay is noticeably greater than half. When the elapsed time is greater than half the fundamental period (about $2 / 3$ ), the emissions rapidly increase their amplitude, whereas when the elapsed time is less than half (about $1 / 3$ ), the emissions rapidly decay and disappear. When the elapsed time is half the fundamental period, the stronger of the emission sets dominates and endures, while the weaker set is soon killed. In addition, three-phase emissions may be observed continuously for several minutes, while the appearance of two phase emissions is in all cases transitory. All these observations may be interpreted as indicating the existence of a relaxation phenomenon in the generation of VLF emissions. It is suggested that after the generation of an emission, some time is required for the medium to recover so that strong emission is again possible. We may think of this phenomenon in terms of the "available" energy in the medium, i.e., available for conversion into VLF emissions. The generation of an emission then "de-energizes" the medium, and some time is required to "pump up" the available energy and thus "prime" the medium 
so that maximum emission is again possible. Of course, the generation of a strong emission deenergizes the medium more than a weak emission.

For this interpretation, it would appear that the rate at which energy is pumped into the medium may be modulated part of the time with a period of about 30 sec. When the pumping rate is high, the average amplitude of the emissions is large, and the recovery time smaller, so that new emissions may be initiated after two-thirds of the fundamental (4 sec) emission period. When the pumping rate is small, no new sets of emissions are initiated, and the average amplitude of the existing emissions is small.
Some additional support for this concept is found in the observation that the individual emissions of the three-phase emissions are noticeably weaker than the single phase emissions. While it is realized that the same quantum-mechanical principles are not applicable here, an analogy with a laser may be considered, in that the emitter is "pumped up" and then "fired" by the introduction of a suitable signal into the system. For a given pumping rate, a higher duty cycle (3 phase-emissions) will result in the individual bursts of radiation being less intense.

The explanation of the stability of the three-phase emissions and the instability of two-phase emissions

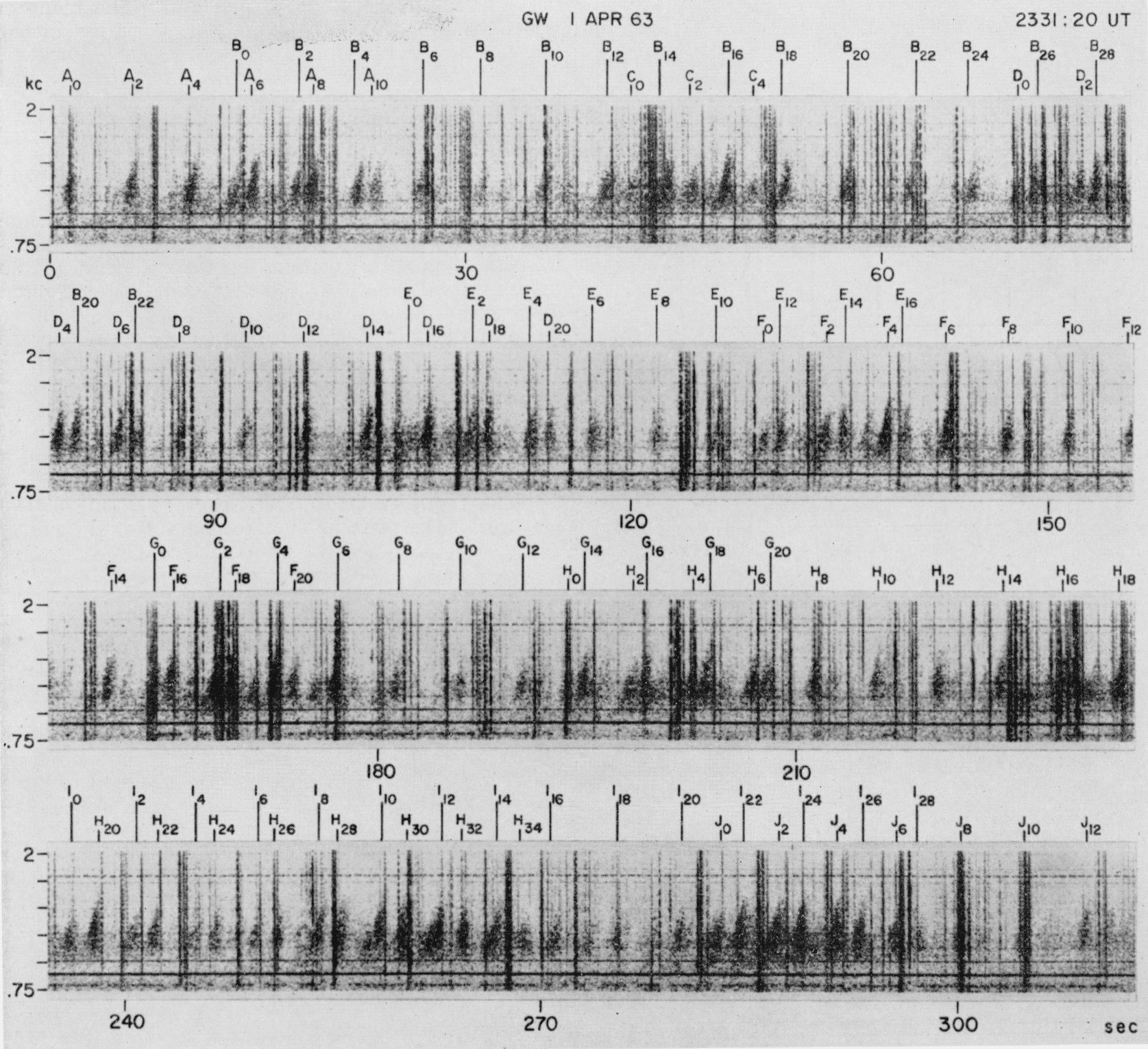

FIGURE 9. The characteristic pattern of two phase emissions showing the growth and decay of sets of emissions. 


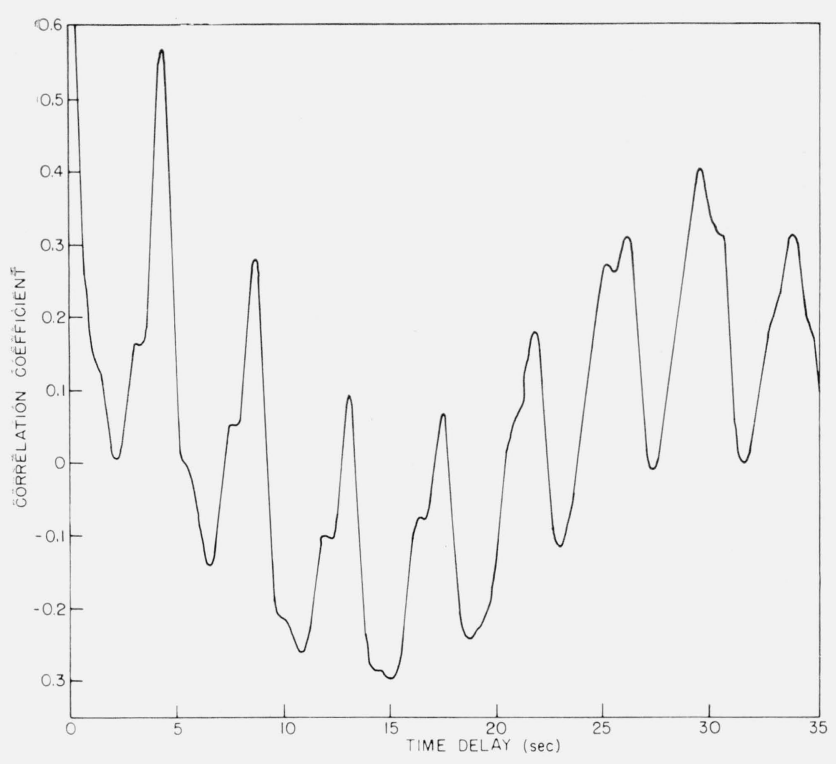

Figure 10. Autocorrelation of the $1.25 \mathrm{kc} / \mathrm{s}$ amplitude of the emissions shown in figure 9.
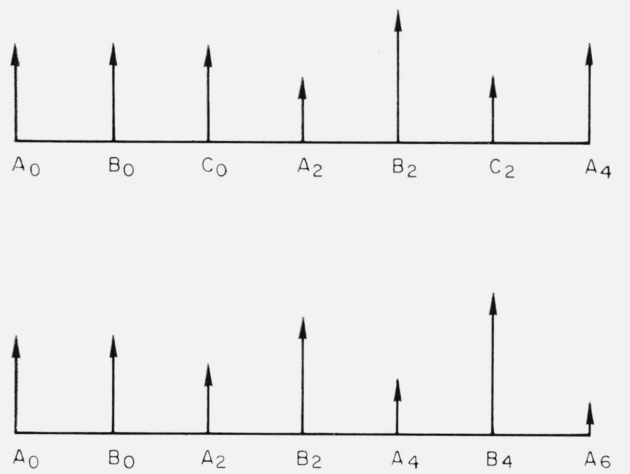

Figure 11. Illustrating the inherent stability of an odd number of phases and instability of an even number.

is illustrated in figure 11 . We have observed that the amplitude of an emission may vary inversely with the amplitude of the previous emissions. Suppose we have initially two emissions, $A_{0}$ and $B_{0}$, equal in amplitude and separated in time by half the fundamental period, and that the emission $A_{2}$ is weakened by some random effect. This may lead to an increase in the amplitude of $\mathrm{B}_{2}$, which in turn gives a weakening of $A_{4}$, which strengthens $B_{4}$ and so on, so that one set of emissions rapidly dominates. Consider now the situation for three sets of emissions, symmetrically spaced, in which $\mathrm{A}_{0}, \mathrm{~B}_{0}$, and $\mathrm{C}_{0}$ are equal in amplitude. Then if $\mathrm{A}_{2}$ is weakened by some means, $B_{2}$ will be strengthened and $C_{2}$ weakened, so that $\mathrm{A}_{4}$ initially weakened, may recover to full amplitude. In this picture, an even number of phases gives "positive feedback," while an odd number gives "negative feedback."

\section{Discussion}

The "relaxation time" in the generation process is reminiscent of a relaxation oscillator suggesting a generation mechanism akin to an oscillator. The only mechanism suggested for the generation of VLF emissions which has this property is the nonconvective plasma instability suggested by Brice [1963] and investigated by Bell and Buneman [1964]. The existence of this instability requires an adequate ratio of the transverse to longitudinal temperatures for the energetic particles [Bell and Buneman, 1964 ; Brice, 1964b].

If the emissions were, in fact, generated by this mechanism, then the long term periodic amplitude modulation could be explained as arising from periodic compression and expansion of the medium which could give rise to a periodic variation in the temperature ratio above [Brice, 1964c]. However, no micropulsations with $30 \mathrm{sec}$ periods were observed at Great Whale River when these emissions were recorded [C. S. Wright, 1964, private communication].

It should be noted that, when VLF emissions were observed to be triggered by morse code transmissions from station NAA, no evidence for a relaxation time was found [Helliwell et al., 1 64]. For the transmission of an "O", comprising three dashes at 200 msec intervals, it was found that each dash triggered an emission. Thus any relaxation time would have to be smaller than $200 \mathrm{msec}$ on this occasion, whereas for the emissions discussed above, the characteristic relaxation period appears to be of the order of a few seconds. However, the emissions triggered by manmade signals appear to be unusual in several other respects, and the absence of any evidence of a relaxation phenomenon for them is not considered as a serious objection to this interpretation of the features of the periodic emissions discussed above

The author acknowledges his indebtedness for generous assistance from all his coworkers at the Radioscience Laboratory, Stanford University, and in particular from Drs. R. L. Smith, R. A. Helliwell, and B. Lusignan.

This work was sponsored in part by the Air Force Office of Scientific Research under grant AFOSR 62-370; by the Office of Antarctic Programs of the National Science Foundation under grants NSF-G23875 and NSF-GA-56; by the Atmospheric Sciences Section of the National Science Foundation under grant NSF-GP-1191; and by the Office of Computer Sciences in the Mathematical Division of the National Science Foundation under grant NSFGP948.

\section{References}

Bell, T. F., and O. Buneman (March 2, 1964), Plasma instability in the whistler mode caused by a gyrating electron stream, Phys. Rev. 133, No. 5A, A1300-A1302. 
Brice, N. M. (November 1962), Discussion of paper by R. L. Dowden, doppler-shifted cyclotron radiation from electrons: a theory of very low frequency emissions from the exosphere, J. Geophys. Res. 67, No. 12, 4897-4899.

Brice, N. M. (August 1, 1963), An explanation of triggered VLF emissions, J. Geophys. Res. 68, No. 15, 4626-4628.

Brice, N. M. (1964a), Electron density and path latitude determination from VLF emissions, J. Atmos. Terr. Phys. (in press).

Brice, N. M. (1964b), Fundamentals of VLF emission generation mechanisms, J. Geophys. Res. 69, No. 21, 4515-4522.

Brice, N. M. (1964c), A qualitative explanation of the diurnal variation of chorus, J. Geophys. Res. 69, No. 21, 4701-4703.

Carpenter, D. L. (March 15, 1960), Identification of whistler sources on visual records and a method of routine whistler analysis, TR No. 5, Ctr. AF18(603)-126, Radioscience Lab., Stanford Electronics Labs., Stanford University, Stanford, Calif.

Dinger, H. E. (July 1957), Periodicity in dawn chorus, paper presented at IRE-URSI Symposium, May 22-25, 1957, Washington, D.C., Abstr. in IRE Trans. Ant. Prop. AP-5, No. 3, 325 .

Gallet, R. M. (February 1959), The very low frequency emissions generated in the earth's exosphere, Proc. IRE 47, No. 2, 211-231.
Helliwell, R. A. (October 1, 1963), Whistler-triggered periodic VLF emissions, J. Geophys. Res. 68, No. 19, 5387-5395.

Helliwell, R. A., and N. M. Brice (1964), VLF emission periods and whistler-mode group delays, J. Geophys. Res. 69, No. 21, 4704-4708.

Helliwell, R. A., J. Katsufrakis, M. Trimpi, and N. Brice (June 1, 1964), Artificially stimulated very-low-frequency radiation from the ionosphere, J. Geophys. Res. 69, No. 11, 2391-2394.

Lokken, J. E., J. A. Shand, Sir S. C. Wright, K.C.B., O.B.E., and L. H. Martin, N. M. Brice, and R. A. Helliwell (October 1961), Stanford-Pacific Naval Laboratory conjugate point experiment, Nature 192, No. 4800, 319-320.

Pope, J. H., and W. H. Campbell (August 1960), Observations of a unique VLF emission, J. Geophys. Res. 65, No. 8, 2543-2544.

Smith, R. L., and D. L. Carpenter (August 1961), Extension of nose whistler analysis, J. Geophys. Res. 66, No. 8, 2582-2586.

(Paper 69D2-462) 